\title{
Validity and Reliability of the Modified Physical Activity Questionnaire for Adolescents (PAQ-A) among Malaysian Youth
}

\author{
Denise Koh", Nur Hamizah Zainudin, Mohd Khairi Zawi \\ Faculty of Education, University Kebangsaan Malaysia, Malaysia
}

Received August 6, 2020; Revised October 26, 2020; Accepted November 5, 2020

\section{Cite This Paper in the following Citation Styles}

(a): [1] Denise Koh, Nur Hamizah Zainudin, Mohd Khairi Zawi, "Validity and Reliability of the Modified Physical Activity Questionnaire for Adolescents (PAQ-A) among Malaysian Youth," International Journal of Human Movement and Sports Sciences, Vol. 8, No. 6, pp. 355 - 360, 2020. DOI: 10.13189/saj.2020.080606.

(b): Denise Koh, Nur Hamizah Zainudin, Mohd Khairi Zawi (2020). Validity and Reliability of the Modified Physical Activity Questionnaire for Adolescents (PAQ-A) among Malaysian Youth. International Journal of Human Movement and Sports Sciences, 8(6), 355 - 360. DOI: 10.13189/saj.2020.080606.

Copyright $\subseteq 2020$ by authors, all rights reserved. Authors agree that this article remains permanently open access under the terms of the Creative Commons Attribution License 4.0 International License

\begin{abstract}
This study aims to measure the level of validity and reliability of Physical Activity Questionnaire for Adolescents (PAQ-A) in Bahasa Malaysia in measuring Malaysian youths' level of fitness. The absence of Bahasa Malaysia questionnaire in measuring the level of Malaysian youths' fitness further strengthens the study carried out. Descriptive statistics are applied to describe respondents' demographic distribution such as sex, race, height, weight and BMI. A total of 60 respondents were involved in this study, 29 of them are males (48.3\%) and 31 others are females $(51.7 \%)$. Reliability of PAQ-A is determined by doing test retest after 12 days. Convergent validity and criterion validity were measured by comparing PAQ-A and IPAQ as well as PAQ-A and pedometer overall pace. Pearson Correlation (r), Alpha Cronbach ( $\alpha$ ), and ICC were used to assess the degree of reliability while Spearman Correlation (rho) was used in deciding level of validity of PAQ-A. Overall correlation between first and second PAQ-A administration is $\mathrm{r}=0.719, \mathrm{p}<0.05$. Coefficient $\alpha$ PAQ-A was 0.836 , with the value of $\mathrm{ICC}=0.718$ and $95 \%$ range $\mathrm{CI}=0.569-0.822$. Correlation value between PAQ-A and pedometer overall pace is low $(\mathrm{rho}=0.167, \mathrm{p}=0.202)$. Meanwhile, the correlation between PAQ-A and IPAQ was high ( $\mathrm{rho}=0.516, \mathrm{p}<0.05)$. In conclusion, PAQ-A questionnaire has high degree of reliability and convergent validity and is acceptable in measuring Malaysian youths' fitness level but is low in measuring criterion validity level.
\end{abstract}

Keywords PAQ-A, Validity and Reliability

\section{Introduction}

The ability to measure physical activity accurately is important in research looking at quantifying physical activity behaviour. This importance is increasing as research has showed the benefits of physical activity and the negative consequences of a sedentary lifestyle for a range of negative health outcomes [1]. Physical activity has been found to have a dose-response relationship with health status [2]. Physical activity has been associated with reduced risk of obesity, cardiovascular disease, type 2 diabetes mellitus, osteoporosis and certain type of cancers $[3,4]$. Therefore, physical activity remains an important tool in health promotion interventions. However, due to the nature of human behaviour, one of the main challenges that researchers must face is measuring physical activity accurately and reliably.

In Malaysia, the prevalence of obesity has increased from $5.7 \%$ in 2011 to $11.9 \%$ in 2015 [5, 6], and the overweight and obese are getting younger. Efforts to overcome this problem have seen more researchers in Malaysia exploring ways to increase physical activity among children and adolescents. Although current trend in physical activity research is moving towards objective 
measures using accelerometers, this method comes with its unique challenges, which in turn maintains a need to have a valid and reliable instrument to allow for self-reporting [7]. Although data obtained through the administration of physical activity questionnaires may have its biases, the advantages of using a questionnaire include cost effectiveness, ease of administration.

Self-reported questionnaires commonly used in research with adolescents includes the Physical Activity Questionnaire for Older Children [8, 9] and the Physical Activity Questionnaire for Adolescents (PAQ-A) [10]. The more widely used questionnaire to measure physical activity behaviour among adolescents is the Physical activity Questionnaire for Adolescents (PAQ-A).

The PAQ-A consists of nine (9) items measuring physical activity behaviour in the last seven days. These items include assessing the type of physical activity the children participate during their free time, their physical activity behaviour during recess time in school, and their daily routine. The measurement of general physical activity is strength of the PAQ-A due to the difficulty in precise measurement of intensity, frequency, and duration of young people's activity throughout the day, especially via self-report [10]. The most valuable strength of the PAQ-A is the cost effectiveness, time efficiency and ease of administration in a large-scale study, and the display of normal distribution properties.

However, as with the study of human behaviour, the patterns of physical activity behaviour and the accuracy of self-report may be influenced by gender, cultural or ethnic backgrounds. Thus, it is important for researchers to use valid and reliable instrument for each population, and many researchers have adapted established questionnaire to be used in their population [11-15]. Testing the validity and reliability of a modified PAQ-A for the Malaysian population will allow researchers to have a comparable self-report instrument in which to measure physical activity among adolescents.

Therefore, the aim of this study is to test the validity and reliability of a modified physical activity questionnaire that is suitable to use in the Malaysian adolescent population. Modification of the PAQ-A includes language and activities modifications. Since the writing of this paper, no known studies have tested the validity and reliability of a modified PAQ-A for the Malaysian population.

\section{Materials and Meth5ods}

\section{Cultural Adaptations}

The PAQ-A was originally developed in English and targeted at adolescents. The process to adapt the PAQ-A to the Malaysian adolescents includes two aspect, through language adaptation and context adaptations. PAQ-A was translated from English to the Malay Language by certified translator. The translated Malay Language version of
PAQ-A were then translated back into the English Language by a different certified translator. The translated English version of PAQ-A were then compared with the original version to check for deviation of meaning for each item. Once the two English Language versions of PAQ-A were assessed to have the same meaning, the version of the Malay Language PAQ-A used for the final language translation was deemed finalized and used in this study.

The PAQ-A was also modified to reflect the Malaysian society, where the list of sports included in item 1 were modified to reflect the sports most played by Malaysian adolescents. For example, sports like floor hockey, street hockey, ice hockey, ice skating, and rowing were replaced by futsal, handball, netball and cricket.

\section{Anthropometry Measurements}

Weight and height were measured using the standard protocol from the SEGAK test (KPM, 2016). All measurements were recorded to the nearest two decimal points. Body mass composition were then calculated based on these measurements, and categorized into underweight $(<18.5)$, normal weight $(18.5-23.0)$, overweight $(23.1$ 27.4) and obese $(\geq 27.5)$ according to the WHO recommended cut-offs for Asian population [16].

\section{Physical Activity Measurements}

Physical activity was measured using two self-reported instruments (IPAQ and PAQ-A) and pedometer. PAQ-A has nine (9) items where items 1 to 8 were scored from 1 to 5 , depending on the responses. An average composite score for PAQ-A was then calculated for items 1 to 8 excluding item 9, where a score of 1 indicates low physical activity and a score of 5 indicates high physical activity.

To assess construct validity, the short form International Physical Activity Questionnaire (IPAQ, Ainsworth, et al, 1993) and pedometers were used to measure physical activity. The IPAQ has seven (7) items measuring the estimated time walking, doing moderate to vigorous physical activity for weekdays and weekends. Metabolic equivalent (MET) values for walking (3.3 METs), moderate-intensity physical activity (4.0 METs) and vigorous-intensity physical activity (8.0 METs) were used to calculate walking MET-minutes/week, moderate MET-minutes/week, vigorous (VPA) MET-minutes/week (Ainsworth, et al, 1993; Ainsworth et al, 2000). Total moderate-intensity physical activity (MPA) MET-minutes/week was calculated by summing up walking and moderate-intensity MET-minutes/week. Total moderate- and vigorous-intensity physical activity (MVPA) MET-minutes/week was calculated by summing up all three MET-minutes/week. Participants were also asked to wear a pedometer for one 24-hours day, during a school day.

\section{Procedure}

Adolescents aged 15 to 17 were recruited via their 
schools to participate in this study. Study information sheet and consent form were given to the identified students and told to pass it to their parents. Students were given a week to return the signed consent form. Students whose parents have consented to their participating in this study were then given a questionnaire to fill in. The self-administered questionnaire contained both PAQ-A and IPAQ items, as well as basic demographic information.

They were instructed to complete the questionnaire in class, with the researchers on hand to guide when necessary. After two weeks, the students were then given a pedometer to wear. Instructions of the care and protocol for the pedometers were explained in detail to each student. Students need to show basic understanding on the use and protocol for the pedometer before being allowed to wear the pedometer. Pedometers were worn for one 24-hour day. Students were again administered the PAQ-A and IPAQ in a one week interval. The immediate administration of the PAQ-A and IPAQ after the 7-days of wearing the pedometer will allow the data of the second administered PAQ-A to measure the same time frame as with the pedometer.

\section{Statistical Analysis}

Construct validity was assessed using Spearman Correlation analysis comparing physical activity behaviour data between PAQ-A and step count. Convergent validity was assessed by conducting Spearman Correlation analysis between PAQ-A and IPQA. Internal consistency reliability was also measured using Cronbach's alpha coefficient. Test-retest reliability was assessed by conducting Spearman Correlation analysis between PAQ-A data obtained during the first and second administration of the questionnaire. Data distribution was tested for normality using the Kolmogorov-Smirnov test. Comparison of physical activity behaviour between gender and ethic groups was conducted using the two-independent t-test. Homogeneity of variance was confirmed using the Levene's test.

\section{Results}

A total of 60 consent form was received and the students were included in this study. Participants in this study has similar proportions between male and female (male: $n=29$, $48.3 \%$ and female: $n=31,51.7 \%$ ). The Malay ethnic group was most represented $(81.7 \%)$ in this sample, with the majority $(46.7 \%)$ being underweight $(16.5 \pm 1.19)$ and normal weight $(35.0 \%)$, as shown in Table 1.

\section{Validity}

The distribution scores for PAQ-A were normally distributed for the first $(\mathrm{D}(60)=0.083, \mathrm{p}=0.20)$ and second $(\mathrm{D}(60)=0.88, \mathrm{p}=0.20)$ administrations. However, all other measures of physical activity data were not normally distributed. Overall PAQ-A scores was not significantly correlation with step count $(\mathrm{r}(58)=0.23, \mathrm{p}=0.076)$.

Convergent validity was measured by comparing the scores between PAQ-A and IPAQ MET-minutes/week for moderate-intensity, MET-minutes/week vigorous-intensity and MET-minutes/week total physical activity (see Table 2). This study found that there were significant association between the first administration of PAQ-A scores with MPA ( $r s=0.259, p=0.046)$, VPA ( $r=0.370, p=0.004)$ and MVPA ( $r s=0.370, p=0.004$ ), and the second administration of PAQ-A scores with MPA ( $\mathrm{rs}=0.459, \mathrm{p}<0.001)$, VPA ( $\mathrm{rs}=0.482, \mathrm{p}<0.001)$ and MVPA $(\mathrm{rs}=0.516, \mathrm{p}<0.001)$, as shown in Table 2.

Table 1. Demographic characteristics of the respondents

\begin{tabular}{cc}
\hline Demographic & $\mathbf{N}(\%)$ \\
\hline Gender & $29(48.3 \%)$ \\
Boys & $31(51.7 \%)$ \\
Girls & \\
Ethnicity & $49(81.7 \%)$ \\
Malay & $11(18.3 \%)$ \\
Others & \\
& \\
Body mass index & $28(46.7 \%)$ \\
Underweight & $21(35.0 \%)$ \\
Normal & $4(6.7 \%)$ \\
Overweight & $7(11.7 \%)$ \\
Obese & \\
\hline
\end{tabular}

Table 2. Correlation of IPAQ walking MET-minutes/week, moderate-intensity MET-minutes/week and vigorous-intensity MET-minutes/week with PAQ-A scores for time-1 and time-2 administration

\begin{tabular}{|c|c|c|}
\hline & $\begin{array}{c}\text { Mean } \pm \text { sd } \\
\begin{array}{c}\text { Median (interquartile } \\
\text { range) }\end{array} \\
\end{array}$ & p-value \\
\hline \multicolumn{3}{|l|}{ TIME-1 } \\
\hline PAQ-A & $2.64 \pm 0.68$ & - \\
\hline \multicolumn{3}{|l|}{ IPAQ } \\
\hline $\begin{array}{c}\text { MPA } \\
\text { MET-minutes/week }\end{array}$ & $1297.50(786.00-2862.00)$ & $0.046^{*}$ \\
\hline $\begin{array}{c}\text { VPA } \\
\text { MET-minutes/week }\end{array}$ & $1440.00(720.00-2790.00)$ & $0.004 *$ \\
\hline $\begin{array}{c}\text { MVPA } \\
\text { MET-minutes/week }\end{array}$ & $3128.00(1809.98-5781.75)$ & $0.004 *$ \\
\hline \multicolumn{3}{|l|}{ TIME-2 } \\
\hline PAQ-A & $2.64 \pm 0.67$ & - \\
\hline \multicolumn{3}{|l|}{ IPAQ } \\
\hline $\begin{array}{c}\text { MPA } \\
\text { MET-minutes/week }\end{array}$ & $1518.00(739.75-3274.50)$ & $<0.001 *$ \\
\hline $\begin{array}{c}\text { VPA } \\
\text { MET-minutes/week }\end{array}$ & $880.00(390.00-2060.00)$ & $<0.001^{*}$ \\
\hline $\begin{array}{c}\text { MVPA } \\
\text { MET-minutes/week }\end{array}$ & $2760.75(1608.75-5274.98)$ & $<0.001 *$ \\
\hline
\end{tabular}

\section{Test-Retest Reliability}

The Pearson Correlation (r) was conducted to measure 
the relationship between the first and the repeated PAQ-A scores. The overall PAQ-A scores between the first and second administration of the questionnaire did not differ much (see Table 2), and have a significant correlation $(\mathrm{r}(58)=0.719, \quad \mathrm{p}<0.001)$. All individual items were significantly correlated between the first and repeated administration of the PAQ-A. Overall PAQ-A scores were also highly positively correlated $(r=0.72, p<0.001)$. This result shows that the Malay version of the PAQ-A has high test-retest reliability (see Table 3).

Table 3. Correlation Coefficient of PAQ-A scores between the first and second administration of the questionnaire

\begin{tabular}{ccc}
\hline Items & Pearson Correlation $(\mathbf{r})$ & $\mathbf{p}$ \\
\hline 1 & 0.84 & $<0.001^{*}$ \\
2 & 0.47 & $<0.001^{*}$ \\
3 & 0.34 & $0.008^{*}$ \\
4 & 0.58 & $<0.001^{*}$ \\
5 & 0.46 & $<0.001^{*}$ \\
6 & 0.54 & $<0.001^{*}$ \\
7 & 0.48 & $<0.001^{*}$ \\
8 & 0.69 & $<0.001^{*}$ \\
\hline Total & 0.72 & $<0.001^{*}$ \\
\hline
\end{tabular}

Table 4 below shows the Alpha Cronbach $(\alpha)$, interclass correlation coefficient-ICC and 95\% Confidence Interval. There were significant correlation between the first and second administration and demonstrated good internal consistency.

Table 4. Alpha Cronbach, ICC and 95\% CI between PAQ-A (administration 1) and PAQ-A (administration 2)

\begin{tabular}{cccc}
\hline Items & Alpha Cronbach $(\boldsymbol{\alpha})$ & ICC & $\mathbf{9 5 \%} \mathbf{C I}$ \\
\hline 1 & 0.91 & 0.84 & $0.75-0.90$ \\
2 & 0.64 & 0.47 & $0.25-0.65$ \\
3 & 0.51 & 0.34 & $0.09-0.54$ \\
4 & 0.73 & 0.58 & $0.38-0.72$ \\
5 & 0.63 & 0.45 & $0.23-0.63$ \\
6 & 0.70 & 0.54 & $0.33-0.69$ \\
7 & 0.65 & 0.48 & $0.26-0.65$ \\
8 & 0.81 & 0.69 & $0.53-0.80$ \\
\hline Total & 0.84 & 0.72 & $0.57-0.82$ \\
\hline
\end{tabular}

\section{Physical Activity Behaviour}

The overall physical activity level of adolescent in this study were low for both time-1 $(\mathrm{M}=2.64 \pm 0.69)$ and time-2 $(\mathrm{M}=2.64 \pm 0.67)$. This study found that boys were significantly more active compared to girls in PAQ-A scores for both time-1 $(\mathrm{t}(58)=4.21,95 \% \mathrm{CI}=0.34$ to 0.97$)$ and time-2 $(\mathrm{t}(58)=3.18,95 \% \mathrm{CI}=0.19$ to 0.83$)$. However, the association between ethnic group and physical activity is not that strong. Only PAQ-A scores collected in time-2 showed that Malays were significantly less active compared with the Chinese and Indian ethic group $(\mathrm{t}(58)=$ $-2.051,95 \% \mathrm{CI}=-0.96$ to -0.11 ).

Table 5. Comparison of physical activity status (PAQ-A) between gender and ethnic group

\begin{tabular}{|c|c|c|c|c|c|}
\hline & $\mathbf{N}$ & $\begin{array}{c}\text { TIME-1 } \\
(\text { Mean } \pm \text { sd })\end{array}$ & p-value & $\begin{array}{c}\text { TIME-2 } \\
(\text { Mean } \pm \text { sd })\end{array}$ & p-value \\
\hline \multicolumn{6}{|l|}{ Gender } \\
\hline Boys & 29 & $2.98 \pm 0.67$ & $<0.001^{*}$ & $2.91 \pm 0.67$ & $0.002 *$ \\
\hline Girls & 31 & $2.33 \pm 0.54$ & & $2.37 \pm 0.57$ & \\
\hline \multicolumn{6}{|l|}{ Ethnic Group } \\
\hline Malay & 49 & $2.57 \pm 0.69$ & 0.106 & $2.55 \pm 0.63$ & $0.015^{*}$ \\
\hline $\begin{array}{l}\text { Others (Chinese and } \\
\text { Indian) }\end{array}$ & 11 & $2.94 \pm 0.57$ & & $3.08 \pm 0.70$ & \\
\hline
\end{tabular}




\section{Discussion \& Conclusions}

The results of this study found that the Malay language PAQ-A has a fair reliability and acceptable validity to measure physical activity behaviour among adolescents in Malaysia. The PAQ-A Malay Language will be useful for the monitoring of physical activity behaviour among adolescent in Malaysia at the population level.

There were mixed results in the validity testing of the Malay language version of PAQ-A. The study found that the Malay language PAQ-A were significantly correlated with scores from IPAQ. However, the same was not observed between PAQ-A scores and step count. This may be due to the short duration of wearing pedometer in this study. One 24-hour day wear duration was adopted in this study due to the low compliance rate among the students.

While the correlation coefficient reported in this study were small, they are consistent with the psychometric properties reported for the original PAQ-A [10] and by others for self-report questionnaires for physical activity among adolescents (Hong et al, 2012). The Vietnamese study testing the validity of the PAQ-A with IPAQ reported spearmen correlation of 0.25 and 0.22 for the assessment of the questionnaire.

In this study, the correlation found were higher for the data administered during time-2, however, it is important to note that on both administration of the questionnaire, there were significant correlation between PAQ-A with IPAQ for all domain (MPA, VPA and MVPA). Previous studies have reported that low and moderate-intensity physical activities are very difficult for adolescents to recall and report because these activities are accumulated throughout the day and include a myriad of different activities, which makes it very difficult to recall $[19,20]$.

The test-retest reliability correlation coefficient in this study gave similar results as seen in other reliability studies [13]. However, it is important to note that the one-week interval between the administration of test-retest to measure the reliability of PAQ-A may likely have picked up some actual variations in activities between these two time-period, which would have resulted in an underestimation of the reliability coefficient.

The findings where boys were more active compared to girls are consistent with previous studies [21]. The lower rate of participation in PA among girls may be due to the influence of socio-ecological factors at the individual, family, school and environmental levels [21]. These factors are potentially modifiable but will need the collaboration of the whole community to make it happen.

A limitation to this study is the use of pedometers instead of accelerometers. Pedometers are no longer the device of choice to measure physical activity behaviour due to its limitation in measures of acceleration that is used to estimate physical activity intensity levels. The samples for this study were all from urban areas and there were no representation from the rural communities in Malaysia.
Therefore, this study is unable to report on the validity and reliability of Malaysian adolescents in the rural communities.

Recommendations for that future studies looking at the validity and reliability of the PAQ-A to include rural communities so that a more comprehensive assessment of the validity and reliability of PAQ-A Malay Language questionnaire. Future studies should also consider innovative strategies to improve compliance rate of wearing a physical activity measuring device among adolescents. Further, considerations should also be given to using accelerometers instead of pedometer due to the advancing technology of accelerometer as well as the ease of wear of these new technologies.

\section{Conclusions}

In conclusion, this study showed that the PAQ-A Malay Language version may show a good level of convergent validity, but it did poorly for criterion validity. However, this adapted PAQ-A is still a viable alternative to measure physical activity behaviour among Malaysian adolescent at the population level. Further validation in rural communities in Malaysia is needed.

\section{REFERENCES}

[1] DHHS (US Department of Health and Human Services), Physical Activity Guidelines for Americans, 2nd edition. 2018. US: Department of Health and Human Services. https://health.gov/sites/default/files/2019-09/Physical_Activ ity_Guidelines_2nd_edition.pdf (Accessed on 19 Oct 2020).

[2] Warburton D. \& Bredin S.S.D., "Health benefits of physical activity: a systematic review of current systematic reviews," Current Opinion in Cardiology, Vol. 32, no. 5, pp. 541-556, 2017.

[3] Lear S.A., Hu W., Rangarajan S., Gasevic D., Leong D., Iqbal R., Casanova A., Swaminathan S., Anjana R.M., Kumar R., Rosengren A., Li W., Wang Y., Wang C., Liu H., Nair S., Diaz R., Swidon H., Guta R., Mohammadifard N., Lopez-Jaramillo P., Oguz A., Zatonska K., Seron P., Avezum A., Poirier P., Teo K. \& Yusuf S., "The effect of physical activity on mortality and cardiovascular disease in 130000 people from 17 high-income, middle-income, and low-income countries: the PURE study," Lancet, Vol. 390, no. 10113, pp. 2643-2654, 2017.

[4] Wahid A., Manek N., Nichols M., Kelly P., Foster C., Wester P., Kaur A., Smith C.F., Wilkins E., Rayner M., Roberts N. \& Scarborough P. "Quantifying the association between physical activity and cardiovascular disease and diabetes: A systematic review and meta-analysis," Journal of American Heart Association, Vol. 5 pp. e002495, 2016.

[5] Institute of Public Health (IPH), "National Health and Morbidity Survey 2011 (NHMS2011) Vol.2: Non-Communicable Diseases,". Vol.2. Kuala Lumpur; 2011. 
[6] Institute of Public Health (IPH), "National Health and Morbidity Survey 2015 (NHMS2015). Vol. II: Non-Communicable Diseases, Risk Factors \& Other Health Problems," Vol.2. Kuala Lumpur; 2015.

[7] Tremblay, M.S., "Challenges in global surveillance of physical activity," The Lancet Child \& Adolescents Health, Vol. 4 no. 1, pp. 2-3, 2020

[8] Crocker, P. R. E., Bailey, D. A., Faulkner, R. A., Kowalski, K. C., \& McGrath, R., "Measuring general levels of physical activity: Preliminary evidence for the Physical Activity Questionnaire for Older Children," Medicine and Science in Sports and Exercise, Vol. 29, pp. 1344-1349, 1997.

[9] Kowalski, K. C., Crocker, P. R. E., \& Faulkner, R. A., "Validation of the Physical Activity Questionnaire for Older Children," Pediatric Exercise Science, Vol. 9, pp. 174-186, 1997.

[10] Kowalski, K. C., Crocker, P. R. E., \& Kowalski, N. P., "Convergent validity of the Physical Activity Questionnaire for Adolescents," Pediatric Exercise Science, Vol. 9, pp. 342-352, 1997.

[11] Wyszynska J., Matlosz P., Podgorska-Bednarz J., Herbert J., Przednowek K., Baran J., Deren K., Maruz A., "Adaptation and validation of the Physical Activity Questionnaire for Adolescents (PAQ-A) among Polish adolescents: cross-sectional study," BMJ Open, Vol. 9 no. 11 pp. e030567, 2019.

[12] Helou K., El Helou N., Mahfouz M., Mahfouz Y., Salameh P., Harmouche-Karaki M., "Validity and reliability of an adapted arabic version of the long international physical activity questionnaire," BMC Public Health. Vol. 18, no. $1: 49,2017$

[13] Chu A.H., Moy F.M., "Reliability and validity of the Malay International Physical Activity Questionnaire (IPAQ-M) among a Malay population in Malaysia," Asia Pac J Public
Health, Vol. 27, no. 2, pp. NP2381-2389, 2015.

[14] Delshad M., G.A., Ghaleh N.R., Amirshekari G, Askari S., Azizi F, Reliability and validity of the modified activity questionniare for an Iranian Urban Adolescent Population. International Journal of Preventive Medicine, 2015. 6.

[15] Oyeyemi A.L., Bello U.M., Philemon S.T., Aliyu H.N., Majidadi R.W., Oyeyemi A.Y., "Examining the reliability and validity of a modified version of the International Physical Activity Questionnaire, long form (IPAQ-LF) in Nigeria: a cross-sectional study," BMJ Open, Vol. 4, no. 12, pp. : e005820, 2015.

[16] World Health Organisation, "Global Strategy on diet, physical activity and health. Resolution of the fifty seventh World Health Assembly," WHA57.17, 2004.

[17] Washburn R.A., Heath G.W., Jackson A.W., "Reliability and validity issues concerning large-scale surveillance of physical activity," Research Quarterly in Exercise and Sport, Vol. 71 no. 2 Suppl, ppt S104-S113, 2000.

[18] Bassett D.R., "Validity and reliability issues in objective monitoring of physical activity," Research Quarterly in Exercise and Sport., Vol.71, no. 2 Suppl, pp: S30-S36.

[19] Booth M.L., Okely, A.D., Chey T. N., Bauman A., "The reliability and validity of the Adolescent Physical Activity Recall Questionnaire," Medicine and Science in Sports and Exercise, Col. 34, no. 12, pp. 1986-1995, 2002.

[20] Barbosa N., Sanchez C.E., Vera J.A., Perez W., Thalabard J.C., Rieu M., "A physical activity questionnaire: reproducibility and validity," Journal of Sports Science \& Medicine, Vol. 6 no. 4, pp. 505-518, 2007.

[21] Telford R. M., Telford R. D., Oliv, L. S., Cochrane T., \& Dave, R., "Why are girls less physically active than boys? Findings from the LOOK longitudinal study," PloS one, Vol. 11, no. 3, 2016. 